Safa Jida*, Hassan Ouallal, Brahim Aksasse, Mohammed Ouanan, Mohamed El Amraoui and Mohamed Azrour

\title{
Clay-Based Brick Porosity Estimation Using Image Processing Techniques
}

https://doi.org/10.1515/jisys-2018-0191

Received April 21, 2018; previously published online February 23, 2019.

\begin{abstract}
This work intends to apprehend and emphasize the contribution of image-processing techniques and computer vision in the treatment of clay-based material known in Meknes region. One of the various characteristics used to describe clay in a qualitative manner is porosity, as it is considered one of the properties that with "kill or cure" effectiveness. For this purpose, we use scanning electron microscopy images, as they are considered the most powerful tool for characterising the quality of the microscopic pore structure of porous materials. We present various existing methods of segmentation, as we are interested only in pore regions. The results show good matching between physical estimation and Voronoi diagram-based porosity estimation.
\end{abstract}

Keywords: 68U10, 92E99, 68W40.

\section{Introduction}

The structural ceramic tiles and clay materials industry is an important regional industrial segment consuming an important quantity of raw materials, especially clay. Morocco is among the world's top 20 producers and consumers of clayey building materials. The industry of ceramic tiles and bricks is most predominant in northern central Morocco. Clayey materials are plentifully used in this region; they take an important part in the national economy, with a production of about $45 \%$ for building materials (bricks, ceramic tiles and refractories).

Many factors can affect the quality of the clay-based material, for example density, porosity, pore size, permeability and mechanical strength. The effects of porosity on the physical properties of clay have been demonstrated in many studies on sintering and in development programs designed to establish production.

Physics have provided various techniques to calculate the void. For example, porosity can be estimated by computing volumetric measurements of core samples, or from geophysical logs, which calculate a property of a rock and infer its porosity, or from petrographic image analysis, which evaluates the pore size of a small sample. This field is conducted towards the measurement of porosity in clay samples, because it affords the basic concepts for understanding.

The sophisticated structures of porous materials bring important challenges to characterization techniques. In the field of computer science, due to its non-destructive and deep investigating capability, numerous projects have used imaging methods and image analysis to characterize the behaviour of porous media $[7,11,14,22,31,38]$. These works mainly used computed tomography (CT) to describe and analyse some of

\footnotetext{
*Corresponding author: Safa Jida, Department of Computer Sciences, Asia Team, Laboratory M2I, Faculty of Sciences and Techniques Errachidia, Errachidia 52000, Morocco, e-mail: s.jida@edu.umi.ac.ma. https://orcid.org/0000-0002-6131-8755 Hassan Ouallal and Mohamed Azrour: Department of Fundamental and Applied Chemistry, Laboratory PCM, Faculty of Sciences and Techniques Errachidia, Errachidia, Morocco

Brahim Aksasse and Mohammed Ouanan: Department of Computer Sciences, Asia Team, Laboratory M2I, Faculty of Sciences and Techniques Errachidia, Errachidia 52000, Morocco

Mohamed El Amraoui: Department of Physics, Faculty of Sciences Meknes, Moulay Ismail University, Meknes, Morocco
} 
the most important features of porous media, such as conductivity, permeability and porosity. An example of an application using images is structure quantification [13, 36, 39], where the main focus is to demonstrate the utility of industrial-quality CT scanning and density scaling for driving fractal parameters, including the ability of two-dimensional (2D) scans to represent 3D structures. The results of flow modelling and behaviour analysis inside porous media $[19,33,38]$, where relative permeability is computed directly from a digitised image of the fluid phase, are in agreement with experimentally measured values. The mechanics of porous media $[1,34]$ are also studied via imaging using X-ray tomography, such as shear zone, curved shape, and varying degrees of dilatancy and crack opening (in time and space). The properties of 2D porosity [37] are experimentally investigated to detect the penetration of pores by introducing a new concept, homogeneous dimension minimum, which essentially exploits the difference of the greyscale values between two images at the same pixel $i$. Pore diameter is considered one of the challenging tasks in material characterization. She et al. [30] introduced a new parameter called effective pore diameter calculated from a scanning electron microscopy (SEM) image, the algorithm of which is based on Euclidean distance transform. Another interesting field is 3D representation and reconstruction [4, 8, 29] to identify and analyse the microstructure of materials, and direct simulation of 3D flow in the reconstructed pore space.

In this paper, we consider image-processing techniques and physical experiments together in order to measure the porosity of clay materials. The performances of some of the described methods will be demonstrated by comparing the imaging results with the experimental calculations.

In the rest of the paper, we present the physical analysis method that we have used in order to measure porosity for the clay samples. Next, we describe the imaging method we have used to estimate porosity. Later, we demonstrate the utility of imaging techniques by comparing our results with those found by physical analysis. Finally, we discuss and interpret the obtained results and provide our conclusion.

\section{Physical Analysis Method}

In this work, the porosity of the test pieces is determined by the method of impregnation in boiling water according to ISO 10545-3. It is calculated by following the works presented in Refs. [2, 27]. The method can be summarized in the following steps:

- Dry the sample in an oven at $150{ }^{\circ} \mathrm{C}$ for $2 \mathrm{~h}$ or at $110^{\circ} \mathrm{C}$ for $24 \mathrm{~h}$.

- Determine the dry mass $\left(M_{\mathrm{sec}}\right)$.

- Put the samples in a bath of distilled or filtered water and boil them for $5 \mathrm{~h}$, taking in consideration that the specimens are covered with water.

- After boiling, leave the samples for an additional $24 \mathrm{~h}$ in the bath.

- Determine the mass $\left(M_{\mathrm{h}}\right)$ of each sample suspended in water. Perform weighing by placing the specimen in a basket attached to the scale.

- After determining the hanging mass, lightly wipe the sample with a lint-free tissue or cotton tissue to remove any excess water from the surface.

- Determine the saturated mass $\left(M_{\mathrm{sat}}\right)$.

The measurement procedure should be performed as quickly as possible to minimize errors caused by water evaporation from the sample.

Finally, we calculate the porosity by applying Eq. (1):

$$
P=100 \frac{\left(M_{\mathrm{sat}}-M_{\mathrm{sec}}\right)}{\left(M_{\mathrm{sat}}-M_{\mathrm{h}}\right)}(\%)
$$

In total, we have four samples of clay in three different firing temperatures: $S_{0}$ is brut clay; $S_{5}, S_{10}$ and $S_{15}$ are, respectively, three clay samples with $5 \%, 10 \%$ and $15 \%$ of organic matter. The principal objective of adding organic matter is to see its impact on porosity in terms of the firing temperature along with the high mechanical resistance at high temperatures. 


\section{SEM Images}

Here, we describe a non-destructive process using computer vision for porosity estimation. This technique is basically used for characterizing materials such as ceramic. Its basic principle is to give a shot of electron beam to the studied surface. The atoms of the sample release secondary electrons with lower energy, which are processed by a detector that provides the surface micrograph of the sample on a control screen. To observe non-conductive ceramic samples, carbon or platinum metallization is required to make them conductive.

The scanning electron microscope used was FEI QUANTA 200 (FEI, Hillsboro, OR, USA).

\subsection{Image Analysis Method}

Before attempting any kind of treatment, we conduct image enhancement due to the presence of some noise. These fundamental pre-processing steps help the further steps to work well and lead to a successful interpretation. For this purpose, we apply a filter that reduces the noise level.

\subsection{Segmentation Techniques}

Here, we give a description of the segmentation method we have used in investigating the sample used. For measuring the porosity, pore size and pore distribution of the clay, the result of segmentation was used. Segmented images are none other than a binary image. Thus, a whole package of grey-level treatment can be exploited.

\subsubsection{Thresholding Segmentation}

A very simple and fast segmentation and edge detection technique is performed by first creating a binary image through setting an image threshold. The binarization threshold is computed from the grey-level histogram that well separates the class.

This is a prevalent approach to designate classes to the pixels in an image. The idea is based on estimating the global threshold level from the intensity histogram of the image. The pixel classes are attributed by comparing the intensity with this threshold value.

A classic method of this type is described by Otsu [26], where the criterion is to minimize the in-class variance while maximizing the between-class variance. The noise is identified as Gaussian, and an alternative method is to fit a sum of Gaussian functions on the histogram data and use the estimated parameters to determine the optimal threshold levels using decision theory, see e.g. Ref. [21]. Metrics based on the histogram entropy has also been used to identify threshold levels [20]. Finding the threshold in mono-modal histograms, Rosin [28] used a method based on the location of the histogram knee, i.e. the point where the tangent of the main histogram peak meets the tangent of the asymptotic low-probability tail.

Generally, using strict grey-level intervals provided by histogram-based thresholding methods may result in a number of incorrectly classified pixels, which makes these methods inadequate as a final segmentation method. However, histogram-based thresholding is often used as an initial condition for more complex methods, described in the following sections.

\subsubsection{Watershed Segmentation}

The watershed is highly used in $2 \mathrm{D}$ image segmentation. It expresses the image in a geographical term. Its origin is not purely a product of mathematical morphology. It is derived from the geological topology.

This algorithm decomposes the image into homogeneous regions. A greyscale image can be perceived as a topographic relief. The grey level of a pixel in the image can be interpreted as its altitude in the topographic relief. The dark pixels thus correspond to the valleys and basin of the relief, whereas the clear pixels correspond to the hills and ridges. 
One of the difficulties of the implementation of this intuitive analogy is that it gives free choice to its formalization. The watershed algorithm can be implemented in two ways: one is based on recursive immersion algorithm [35] and the other is based on the use of geodesic distance function [25].

Unfortunately, the real images are usually rather noisy when calculating the watershed of their gradient; it shows a strong oversegmentation because the gradient has many minima generated by noise. To overcome this problem, many methods are proposed:

- The first is to filter the original image in order to delete most of the non-significant minima: filtering approach.

- The second is to choose the number of local minima, hence the number of zones we want to highlight with the watershed: swamping approach.

- Finally, we can first apply watershed to obtain a marker for a second application of watershed. As a first result, we obtain a mosaic image. This image is no longer built by pixels but as a planar graph.

\subsubsection{Clustering-Based Segmentation Method}

The clustering-based methods are defined by segmenting images into clusters with pixels sharing the same characteristics. There are two major types of clustering methods: supervised clustering, which needs human interaction to decide the clustering criteria, and unsupervised clustering, which decide on the criteria itself. Each one of these two major techniques includes various approaches (hierarchical $[6,40]$ and density-based methods) that aim for image segmentation.

An example of the best-known method is adaptive $K$-means clustering. The algorithm starts with the selection of $K$ elements from the input data set. The $K$ elements are selected haphazardly from the seeds of clusters. The properties of each element also form the properties of the cluster that is constituted by the element.

\subsubsection{Voronoi Exploitation}

The Voronoi diagram (VD) as a data representation structure has been extensively studied in the field of geometry and applied in different disciplines. Regarding image analysis, the VD arouses a particular interest. In fact, it is an effective way to describe, manipulate and interpret geometric entities. Among the works based on the use of the Voronoi graph, we can cite colour image segmentation $[9,15]$ and texture segmentation [32]. All these works share in common the fact that they apply VD directly on the image itself.

In order to avoid high computational time, several authors $[5,16-18]$ proposed a new approach based on VD using the generated clusters of intensity values given in the vertices of the external boundary of Delaunay triangulation. They applied this method in order to segment face features. In this approach, the authors applied VD on a few selected points $(\leq 255)$ from the 1D image histogram instead of the image itself.

\section{Tests and Results}

In Figure 1, we give the results of segmenting clay SEM images using the method mentioned above. In the raw (B-E), the segmented pores are coloured green, red or white.

For the 12 images, we calculate the percentage of pores existing compared to the whole surface of the image. We obtained a noteworthy result, described in Figure 2.

An important remark to take into consideration is measurement scale; the measurement from the scale bar is respected, as the instruments have been calibrated when it was commissioned.

Figure 2 shows the evolution of porosity in terms of firing temperature. By analysing the results, we can confirm that the sample's porosity is very important towards the lowest temperatures $\left(800{ }^{\circ} \mathrm{C}\right)$ for the different pieces elaborated because the sintering phase is not done well at this temperature [24]. 
From Figure 2, the estimation using VD is close enough to the physical analysis. We can explain the increase in porosity estimation using the imaging method according to the fact of having different types of pores (cavernous) and also fracture samples. Consequently, the SEM image shows them as pores.

Clustering and thresholding methods as seen in Figure 3 give the furthest estimation from the experimental results we can rely on. This can be explained by the fact that $K$-mean segments are not connected and can be scattered very widely. This effect can be reduced by using pixel coordinates as features, an approach that tends to result in large regions being broken up. The thresholding method gives a binary image with a pore/non-pore image, whereas in the SEM image a pore can possess different greyscales.

Comparing Figures 2 and 3, we can notice an important increase in porosity with the introduction of organic matter, as it increases from $13.5 \%$ for the support with $0 \%$ organic matter to $23.5 \%$ with $10 \%$ organic matter at $1000{ }^{\circ} \mathrm{C}$. In parallel, the volume goes from $13.63 \%$ for the support with $0 \%$ organic matter to $35.94 \%$

A
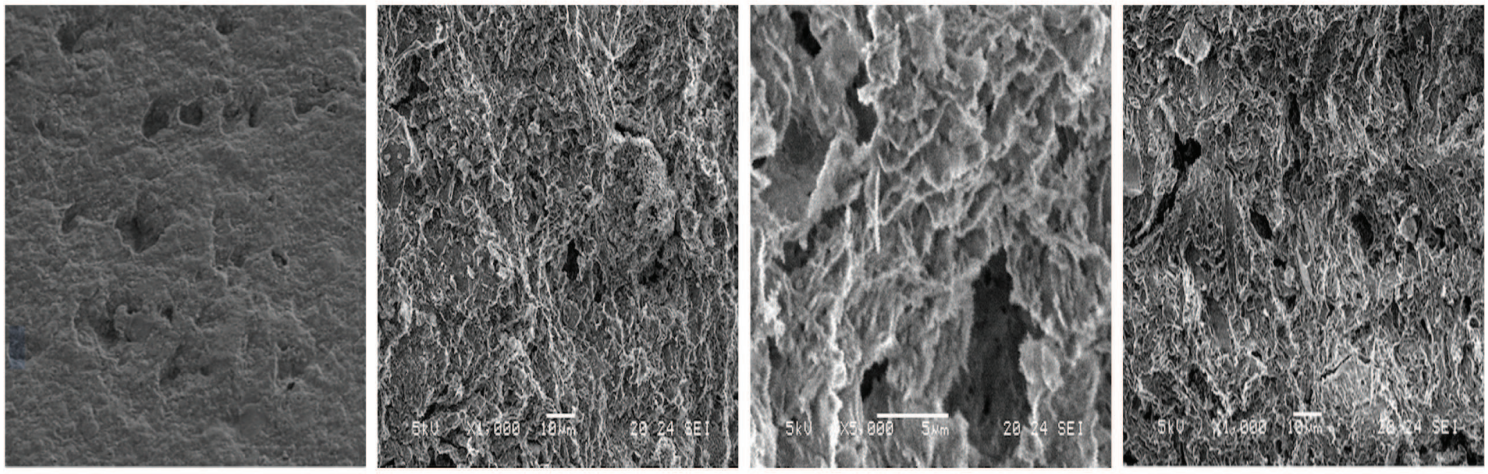

B
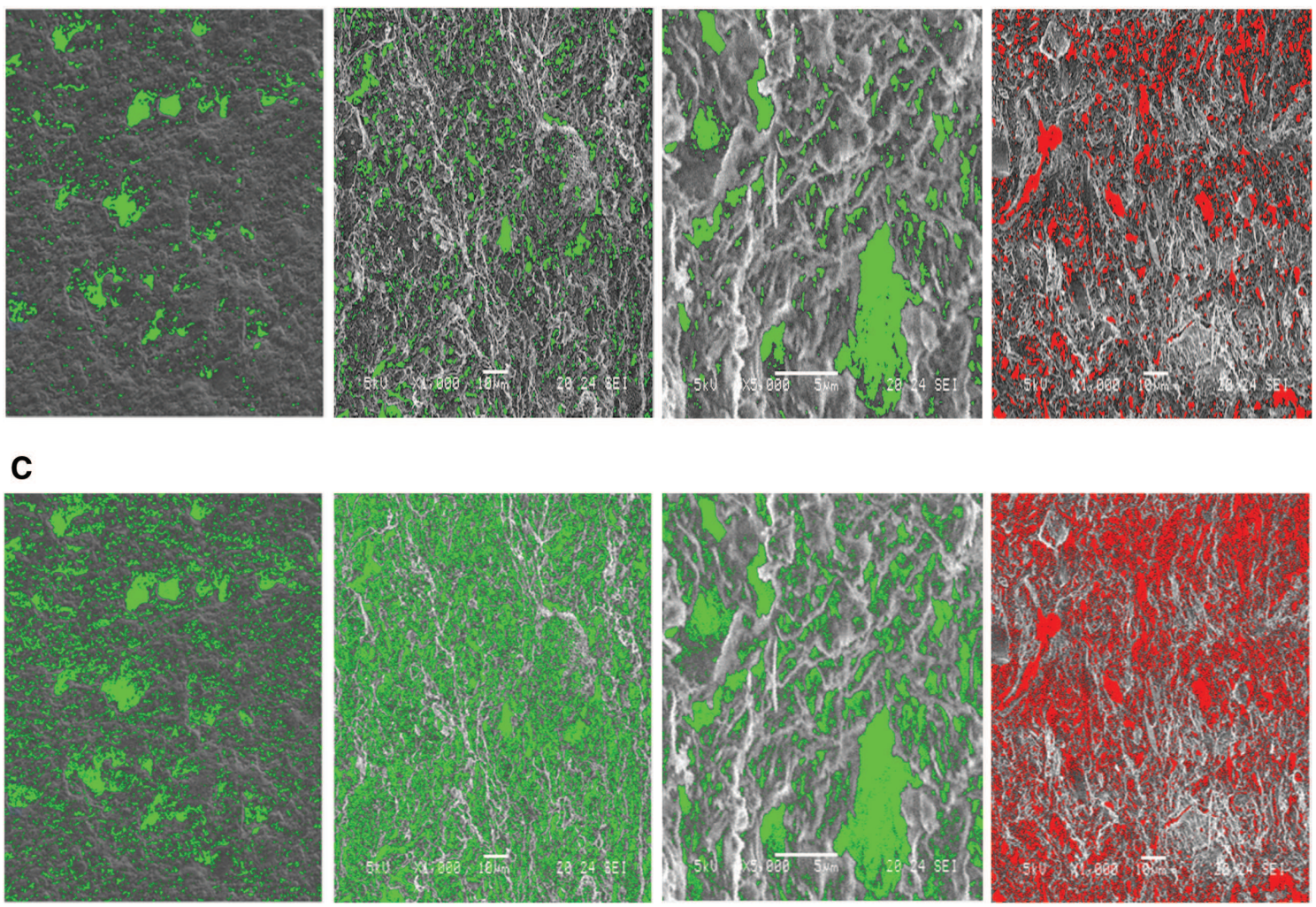

Figure 1: Segmentation Results.

(A) Original images; (B) corresponding VD segmentation; (C) corresponding threshold segmentation; (D) corresponding clustering segmentation; and $(E)$ corresponding watershed segmentation. 
D
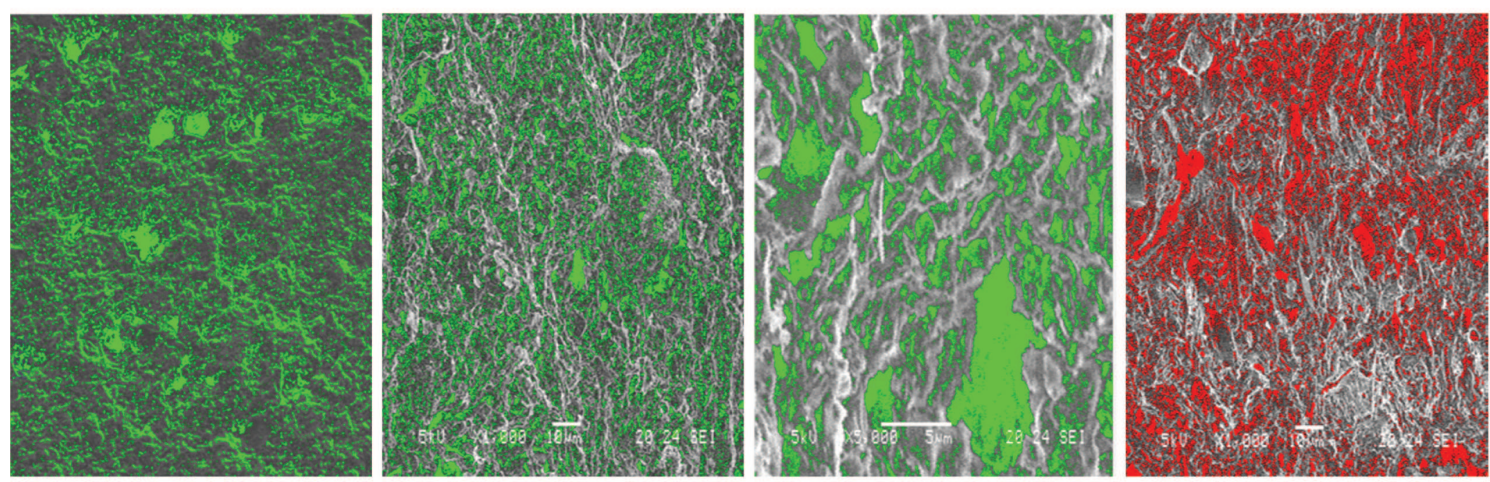

\section{E}
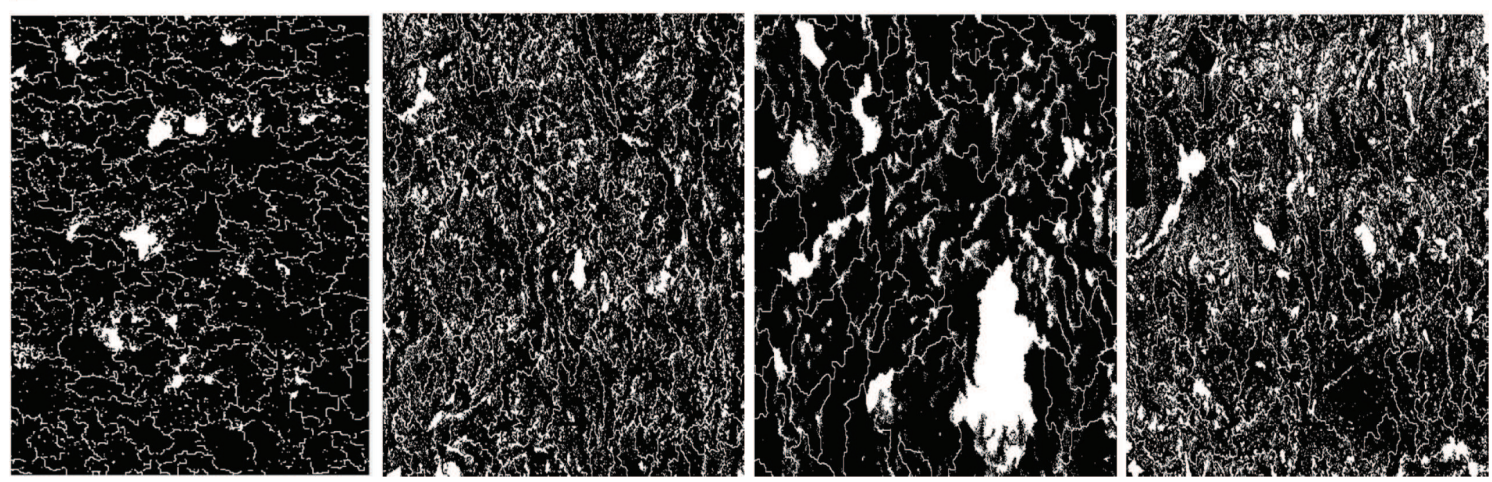

Figure 1 (continued)

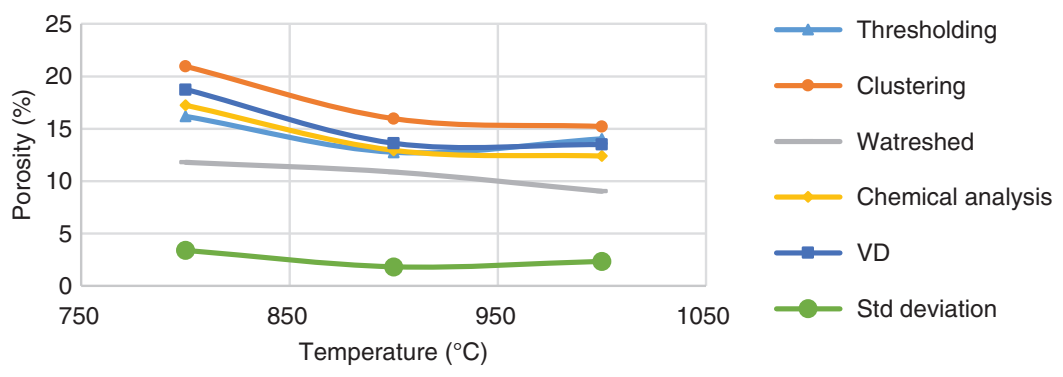

Figure 2: Porosity Estimation-Based VD versus Physical Process for the Sample $S_{0}$.
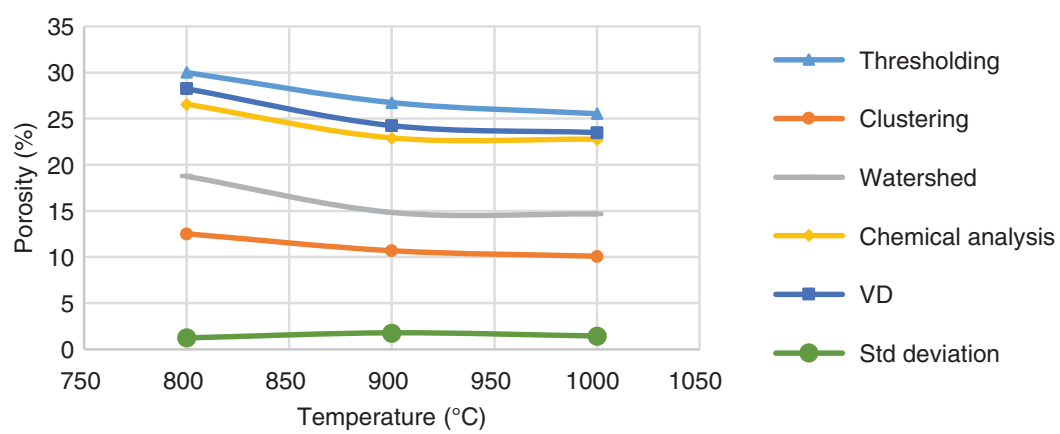

Figure 3: Porosity Estimation-Based VD versus Physical Process for the Sample $S_{10}$. 

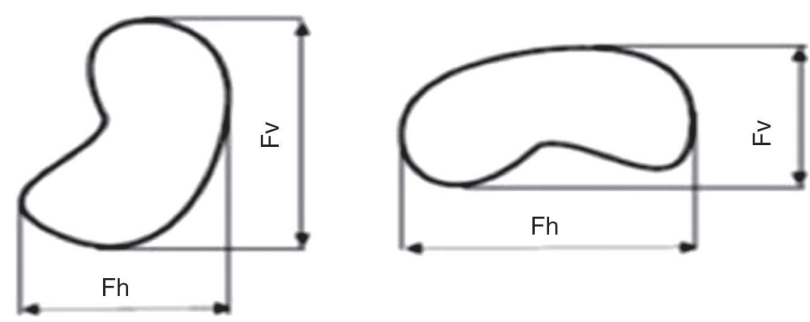

Figure 4: Illustration of Horizontal and Vertical Feret Diameters of a Particle, Fh and Fv, respectively (Wikipedia).

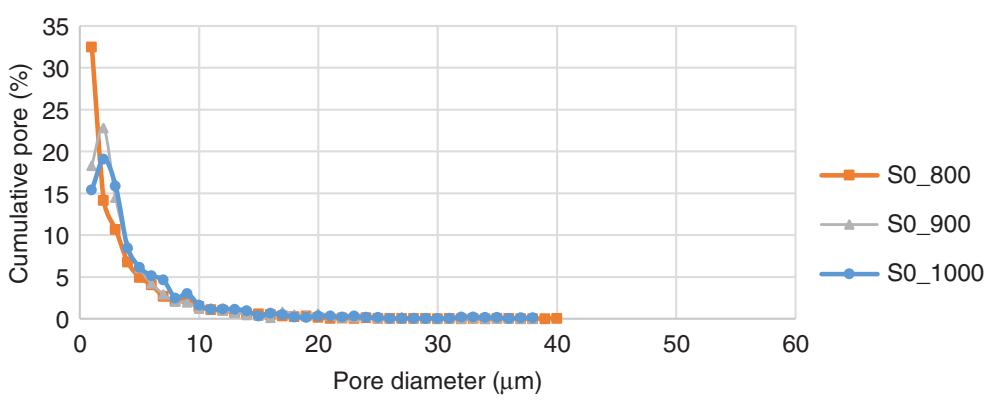

Figure 5: Pore Size Distributions in Raw Clay.

with $15 \%$ organic matter at $1000{ }^{\circ} \mathrm{C}$. At this point, we made sure to keep the porosity as high as possible despite the high firing temperature by adding organic matter.

For the imaging methods we have tested, VD exploitation seems to give a better estimation. The choice of segmentation method should not be done randomly but with suited processing. Recall that the main purpose of any segmentation method is to have an optimal partition based on a given criterion. This requires the definition of the criterion as well as a rule for assigning each point to the appropriate class.

As the pores have an undefined geometric shape, we calculate the pore diameter by using the minimal Feret diameter (Figure 4), which is a measure of an object size along a specified direction. In general, it can be defined as the distance between the two parallel tangents on opposite sides of the image of a randomly oriented particle [23].

In Figure 5, we present the pore distribution of the three temperatures in order to see the effect of firing on the behaviour of the pore.

Analysing the behaviour of the figure, we can notice a decrease in micropore $(<2 \mu \mathrm{m})$ size as long as the firing temperature increases. Some of the particles begin to melt and form a new component, glass, between the others that pull them even closer. A crucial observation is the decrease of pore size from $900{ }^{\circ} \mathrm{C}$, which is in agreement with the expected trend in refractory clay materials in which new crystalline phases develop $[3,10,12]$. The decrease in pore size strengthens the results of low porosity towards high temperature (Figures 2 and 3).

\section{Conclusion}

In this work, we have presented different imaging methods for porosity estimation in clay-based bricks along with physical analysis. The segmentation phase is seen to be crucial for a correct quantitative image analysis of the microstructure. Classical and direct segmentation methods may lead to an inaccurate result, whereas the hybrid method can reform the flaws of the classical method. The hybrid method is seen to be more objective, reliable and economical either in time execution or in physical analysis requirements. An example of the hybrid method we have presented is VD-based segmentation, which seems to give good results compared with other segmentation techniques for porosity estimation. From porosity estimation to pore size distribution, the 
imaging method can be used widely to describe clay-based materials, such as correlating the imaging porosity estimation with some other physical characteristics like mechanical strength and transport properties.

\section{Bibliography}

[1] K. A. Alshibli, S. Sture, N. C. Costes, M. L. Frank, M. R. Lankton, S. N. Batiste and R. A. Swanson, Assessment of localized deformations in sand using X-ray computed tomography, Geotech. Test J. 23 (2000), 274-299.

[2] ASTM C20-00, Standard test methods for apparent porosity, water absorption, apparent specific gravity, and bulk density of burned refractory brick and shapes by boiling water, ASTM International, West Conshohocken, PA, www.astm.org, 2015.

[3] A. Barba, V. Beltran, C. Feliu, J. Garcia, F. Gines, E. Sanchez and V. Sanz, Materias primas para la fabricacion de soportes de baldosas ceramicas (Raw materials for production of ceramic tiles supports), Instituto de Tecnologia Ceramica, Castellon, 2002.

[4] S. Blacher, V. Maquet, F. Schils, D. Martin, J. Schoenen, G. Moonen, R. Jerome and J. P. Pirard, Image analysis of the axonal ingrowth into poly(d,l-lactide) porous scaffolds in relation to the 3-D porous structure, Biomaterials 24 (2003), $1033-1040$.

[5] A. Cheddad, M. Dzulkifli and A. Abd Manaf, Exploiting Voronoi diagram properties in face segmentation and feature extraction, Pattern Recognit. 41 (2008), 3842-3859.

[6] P. Chundi, U. Dayal, M. Sayal and M. Hsu, US20077181678, 2007.

[7] M. Cislerova and J. Votrubova, CT derived porosity distribution and flow domains, J. Hydrol. 267 (2002), 186-200.

[8] J. F. Daian, C. P. Fernandes, P. C. Philippi and J. A. Bellini da Cunha Neto, 3D reconstitution of porous media from image processing data using a multiscale percolation system, J. Petrol. Sci. Eng. 42 (2004), 15-28.

[9] D. Dan, Image segmentation using Voronoi diagram, in: Eighth International Conference on Digital Image Processing (ICDIP 2016), International Society for Optics and Photonics, pp. 100331, Chengdu, China, 2016.

[10] M. Dondi, G. Guarini, M. Raimondo and C. Zanelli, Recycling PC and TV waste glass in clay bricks and roof tiles, Waste Manage. 29 (2009), 1945-1951.

[11] O. Duliu, Computer axial tomography in geosciences: an overview, Earth Sci. Rev. 48 (1999), 265-281.

[12] R. J. Galan-Arboledas, A. Merino and S. Bueno, Use of new raw materials and industrial wastes to improve the possibilities of using ceramic materials from Bailen (Jaen, southern Spain), Mater. Construct. 63 (2013), 553-568.

[13] J. Gibson, H. Lin and M. A. Bruns, A comparison of fractal analytical methods on 2- and 3-dimensional computed tomographic scans of soil aggregates, Geoderma 134 (2006), 335-348.

[14] A. Heijs, C. Ritsema and L. Dekker, Three-dimensional visualization of preferential flow patterns in two soils, Geoderma 70 (1996), 101-116.

[15] R. Hettiarachchi and J. F. Peters, Voronoi region-based adaptive unsupervised color image segmentation, Pattern Recognit. 65 (2016), 119-135.

[16] S. Jida, B. Aksasse and M. Ouanan, Face segmentation and detection using Voronoi diagram and 2D histogram, in: 2017 Intelligent Systems and Computer Vision (ISCV), Fez, pp. 1-5, 2017.

[17] S. Jida, M. Ouanan and B. Aksasse, Color image segmentation using Voronoi diagram and 2D histogram, Int. J. Tomogr. Simul. 30 (2017), 14-20.

[18] S. Jida, B. Aksasse and M. Ouanan, Porosity estimation in carbonate rock based on Voronoi diagram and 2D histogram segmentation in HSV color space, in: Advanced Intelligent Systems for Sustainable Development (Al2SD'2018), vol. 5, Morocco, Tangier, 2018.

[19] A. Kaestner, E. Lehmann and M. Stampanoni, Imaging and image processing in porous media research, Adv. Water Resour. 31 (2008), 1174-1187.

[20] J. N. Kapur, P. K. Sahoo and A. K. C. Wong, A new method for gray-level picture thresholding using the entropy of the histogram, Comput. Vis. Graph Image Process. 29 (1985), 273-285.

[21] S. Kay, Fundamentals of statistical signal processing: detection theory, vol. 2, Prentice Hall, Upper Saddle River, NJ, 1998.

[22] P. Lehmann, P. Wyss, A. Flisch, E. Lehmann, P. Vontobel, M. Krafczyk, A. Kaestner, F. Beck-mann, A. Gygi and H. Flühler, Mapping the structure of porous media using tomography with X-rays from tubes, thermal neutrons and X-rays from synchrotrons: numerical analysis, experiments and applications, Vadose Zone J. 5 (2006), 80-97.

[23] H. G. Merkus, Particle size measurements: fundamentals, practice, quality, p. 15, Springer, Dordrecht, 2012.

[24] L. Messaoudi, Thèse de 3ème cycle, Université Sidi Mohammed ben Abdellah, Faculté des Sciences Dhar el Mehraz, Fès, Maroc, 1987.

[25] F. Meyer, Skeletons in digital spaces, Image Anal. Math. Morphol. 2 (1988), 257-296.

[26] N. Otsu, A threshold selection method from gray-level histograms, IEEE Trans. Syst. Man Cybern. 9 (1979), 62-66.

[27] J. M. Pérez, J. Ma. Rincón and M. Romero, Effect of moulding pressure on microstructure and technological properties of porcelain stoneware, Ceramics Int. 38 (2012), 317-332.

[28] P. L. Rosin, Unimodal thresholding, Pattern Recognit. 34 (2001), 2083-2096. 
[29] E. Sevostianova, B. Leinauer and I. Sevostianov, Quantitative characterization of the microstructure of a porous material in the context of tortuosity, Int. J. Eng. Sci. 48 (2010), 1693-1701.

[30] F. H. She, K. L. Tung and L. X. Kong, Calculation of effective pore diameters in porous filtration membranes with image analysis, Robot. Comput.-Integr. Manufact. 24 (2008), 427-434.

[31] P. Spanne, J. Thovert, C. Jacquin, W. Lindquist, K. Jones and P. Adler, Synchrotron computed microtomography of porous media: topology and transport, Phys. Rev. Lett. 73 (1994), 2001-2004.

[32] M. Tuceryan and K. Jain, Texture segmentation using Voronoï polygons, IEEE Trans. Pattern Anal. Mach. Intell. 12 (1990), 211-216.

[33] M. Turner, L. Knufing, C. H. Arns, A. Sakellariou, T. J. Senden, A. P. Shepparda, R. Sok, A. Limaye, V. Pinczewski and M. A. Knackstedt, Three-dimensional imaging of multiphase flow in porous media, Physica A 339 (2004), 166-172.

[34] G. Viggiani, N. Lenoir, P. Besuelle, M. D. Michiel, S. Marello, J. J. M. Desrues and M. Kretzschmer, X-ray microtomography for studying localized deformation in fine-grained geomaterials under triaxial compression, C. R. Mech. 332 (2004), 819-826.

[35] L. Vincent and P. Soille, Watershed in digital spaces, an efficient algorithm based on immersion simulation, IEEE Trans. Pattern Anal. Mach. Intell. 13 (1991), 583-598.

[36] H. Vogel, I. Cousin and K. Roth, Quantification of pore structure and gas diffusion as a function of scale, Eur. J. Soil Sci. 53 (2002), 465-473.

[37] J. Wang, M. Gan and J. Shi, Detection and characterization of penetrating pores in porous materials, Mater. Character. 58 (2007), 8-12.

[38] D. Wildenschild, J. Hopmans, C. Vaz, M. Rivers, D. Rikard and B. Christensen, Using X-ray computed tomography in hydrology: systems, resolutions, and limitations, J. Hydrol. 267 (2002), 285-297.

[39] A. Yamaji, M. Yokokawa and K. Sao, Analysis of clustering in three-dimensional grain fabric, Geosphere 3 (2007), 108-118.

[40] X. S. Zhou and T. S. Huang, Relevance feedback in image retrieval: a comprehensive review, Multimed. Syst. 8 (2003), 536-544. 